\title{
Uso de las Gonadotropinas para inducir la Ovulación **
}

\author{
Por E. C. Hamblen, M.D., Guy Onhena, M.D.,+ \\ L. S. Massad, M.D., y Francisco García-Conti, M.D.++
}

(Del Departamento de Obstetricia y Ginecología, División de Endocrinología, Duke University Medical Center)

DURHAM, N. C. U. S. A.

La historia de la evolución de la terapéutica gonadotrópica para el tratamiento de los ciclos anovulatorios, es fascinante.

La senda recorrida hasta alcanzar nuestros actuales conceptos, tiene muchas cumbres de injustificado optimismo y muchas depresiones de justificado o injustificado pesimismo.

La historia comienza alrededor del año de 1930.

Puesto que la experiencia de HAMBLEN con esta terapéutica comenzó en el año natal de 1930 y su primer informe publicado apareció en "Endocrinology" (edición de Mayo-Junio) en el año de 1931, el autor principal de este artículo confía en la benevolencia del lector para que se le permita hacer un breve bosquejo histórico.

* Presentado por el autor principal, por invitación, al Congreso Regional de "The International Fertility Association", Acapulco, México, Enero 28-31, 1961, y a la Universidad de Antioquia, Facultad de Medicina, Medellín, Colombia, Febrero 8, 1961.

** Parte de los gastos de estos estudios fueron sufragados por subvención de uno de nosotros $(\mathrm{ECH})$, por el Consejo de Investigación de Duke University y por Ayerst Laboratories, New York, N. Y.

+ Fellow en Endocrinología de la Fundación Fulbright, 1960. Dirección permanente: State University of Ghent, Department of Obstetrics and Gynecology, Pasteurlaan 2, Ghent, Belgium.

++ Fellow en Endocrinología del Ministerio de Salud Pública de Colombia, 1960-1961 Dirección permanente: Pontificia Universidad Católica Javeriana, Departamento de Obstetricia y Ginecología, Bogotá, Colombia, S. A. 


\section{RESUMEN HISTORICO}

Enumeremos algunos de los iniciales e históricos acontecimientos:

1927 - PHILIP SMITH demostró en forma concluyente que la hipofisectomía causa atrofia de las gónadas, de la corteza suprarrenal y de la glándula tiroides.

1928 - ASCHHEIM y ZONDEK demostraron la actividad gonadotrópica de la orina de la mujer embarazada.

1929 - EARL ENGLE demostró que la castración aumenta la actividad gonadotrópica de la pituitaria y puso en duda el origen hipofisario de las gonadotropinas de la orina de la mujer embarazada.

HAMBLEN ha revisado y documentado con muchas referencias, los esfuerzos iniciales de la terapéutica gonadotrópica en dos de sus libros: "Endocrine Gynecology", 1939, y "Endocrinología de la Mujer”, 1945.

Estos primeros empeños fueron obstaculizados por conocimientos fragmentarios aunque progresivos sobre la fisiología endocrina, así como por los métodos insuficientes y con frecuencia poco satisfactorios para la determinación de los niveles cualitativos y cuantitativos de la actividad endocrina en la mujer.

1930-1931 - Las gonadotropinas coriónicas (GC) fueron empleadas por primera vez en el tratamiento de la hemorragia funcional uterina; CAMPBELL y COLLIP, ZONDEK y NOVAK y HURD dieron cuenta sobre el rápido control de este tipo de hemorragia. GEIST y HAMBLEN trabajando independientemente, no observaron una manifiesta hemostasis y posteriormente HAM BLEN estableció la extraordinaria ocurrencia de ovulación cuando a la hemorragia funcional estaban asociados ciclos anovulatorios.

1933-1936 - HAMBLEN estudió los ovarios y endometrios de las mujeres que habían sido tratadas preoperativamente con gonadotropina coriónica, y demostró que la ovulación había ocurrido muy rara vez cuando estaban asociados ciclos anovulatorios. El observó la extraordinaria luteinización de la granulosa y de la teca en los folículos intactos; así mismo notó que cuando había ocurrido ovulación, la función del cuerpo lúteo era estimulada y 
prolongada y ocasionalmente comprobó ovulaciones supernumerarias.

1939 - HAMBLEN declaró: "Las respuestas ováricas a las influencias gonadotrópicas son regidas por tres condiciones generales: a) el origen y carácter de la gonadotropina; b) el estado funcional de la propia pituitaria; y c) la receptividad ovárica”. Estas sentencias todavía actualmente, tienen vigencia.

A manera de corolario, él desarrolló las siguientes deducciones: la terapéutica gonadotrópica no es requerida cuando la función ovárica es normal; esta terapéutica no puede estimular los ovarios en la época de la post-menopausia ni los ovarios que son intrínsecamente incapaces de responder; y el uso lógico de la terapéutica gonadotrópica tiene lugar en el tratamiento de la insuficiencia ovárica debida a una acción hipogonadotrópica de la pituitaria y cuando está asociada a unos cvarios que son capaces intrínsecamente de responder.

La obtención de la gonadotropina equina (EG) alrededor del año de 1938, suscitó esperanzas de que ella podría proveer una terapia efectiva. Pronto HAMBLEN descubrió y reportó que esta gonadotropina, aunque era capaz de producir maduración folicular y como consecuencia, aumento de la producción de estrógenos en ovarios receptivos, no induciría la ovulación.

Por consiguiente, en este estado de conocimientos, los investigadores poseían E'G la cual tenía acción folículo-estimulante y podía producir maduración folicular y estrogenización y CG la cual tenía acción luteinizante y podía prolongar la función del cuerpo lúteo y la consiguiente elaboración de estrógenos y progesterona. Era solamente lógico investigar los usns consecutivos de estas gonadotropinas, confiando que al regular convenientemente las dosis, producirían la ovulación. HAMBLEN ideó tal esquema terapéutico en el año de 1939 y lo denominó "terapia cíclica 1-2 gonadotrópica". Obtuvo así el primer embarazo durante el primer ciclo de este tratamiento, administrado durante la última parte del mes de octubre y la primera del mes de noviembre de 1939. La esterilidad de la paciente, que había transcurrido por espacio de cuatro años de vida conyugal, se debía a ciclos anovulatorios. Este tratamiento afortunado fue presentado en el Congreso de la Asociación Médica del Sur reunido en Memphis, Tennessee, en noviembre de 1939. El producto de este cmbarazo es hoy día un 
simpático joven que sigue estudios universitarios. Esta misma paciente tuvo nuevamente dificultades para lograr una segunda gravidez; se le administraron dos ciclos adicionales de este tratamiento y al segundo ciclo ella logró su embarazo; en resumen, a esta paciente se le administraron tres ciclos de tratamiento y logró quedar embarazada dos veces durante esta terapia. Un informe sobre este primer embarazo obtenido con la terapéutica gonadotrópica así como de otros casos con resultados semejantes, fue publicado en Marzo de 1941 (Am. J. Obst. \& Gynec. 41: 495-510).

Convencido de la bondad de este tratamiento para inducir la ovulación en algunas mujeres y teniendo en cuenta que él había fallado en muchos otros casos, HAMBLEN describió en el año 1945, un método ligeramente complicado de ensayo terapéutico aplicable a mujeres con falla ovárica. El incentivo para esto nació del hecho de las corrientes insuficiencias de diagnóstico etiológico de la falla ovárica.

Se presentó entonces un intervalo de pesimismo respecto de la bondad de la terapéutica gonadotrópica. Aun actualmente hay pocos proponentes de este tipo de terapia. Las razones para este pesimismo eran obvias: por ejemplo, cuando aprendimos a determinar en forma cuantitativa y con exactitud los niveles de la función gonadotrópica, encontramos que muchas pacientes con falla ovárica hipogonadotrópica, no respondían al tratamiento con gonadotropinas; entre éstas, por ejemplo, había un tipo de pacientes que habían sido tratadas para tumores pituitarios, tenían bajos niveles de gonadotropinas, presentaban amenorrea y deseaban tener un embarazo. Se sucedieron entonces más fracasos que éxitos, a pesar de los ensayos de varias clases de tratamiento empleando gonadotropinas solas o en combinación: CG, EG, gonadotropina obtenida de orina de mujeres en la post-menopausia ("Gamone"), gonadotropinas de pituitarias animales obtenidas en mataderos, CG adicionada a un "sinergista pituitario" ("synapoidin"). Haciendo un análisis retrospectivo y a la luz de nuestros conocimientos actuales es posible que algunas de esas fallas puedan ser correlacionadas a estas pacientes que no estaban "en balance" respecto de las funciones suprarrenal y tiroidea. Es también probable que las dosis implicadas fueran o inadecuadas $o$ excesivas y no proporcionadas la una a la otra, cuando fué evaluado el empleo progresivo de las dos gonadotropinas. Hay la posibilidad de que la terapia original cíclica 1-2 gonadotrópica ha- 
bría podido ser favorablemente modificada por la combinación de EG adicionada de CG durante la primera mitad del tratamiento para administrar entonces CG durante su última mitad.

Durante los últimos años ha ocurrido un resurgimiento en el interés sobre la terapia gonadotrópica debido a los estudios experimentales con gonadotropina pituitaria de origen humano-hormona folículo estimulante (HP - FSH), seguida de la administración de CG es decir, que el esquema cíclico 1-2 continúa siendo empleado (GEMZELL et al J. Clin. Endocrinoiogy \& Metab. 18: 1333$1348,1958)$. Obviamente nosotros no podemos confiar en forma demasiado optimista de la obtención de gonadotropina pituitaria humana en cantidades comerciales. Algunas de las proteínas químicas pueden con el tiempo, ser capaces de reproducir una moderada imitación de esta gonadotropina. Por otra parte, parece ser razonable el reconsiderar nuestro interés en la terapia cíclica 1-2 gonadotrópica con EG y CG, haciendo hincapié en el uso de dosis más apropiadas, empleo de posibles combinaciones hormonales y teniendo la seguridad de que las pacientes que se van a beneficiar con esta terapéutica poseen una función suprarrenal y tiroidea normal.

Aunque desde los días iniciales en que la EG fue empleada, se hicieron varias advertencias acerca de la posibilidad de que esta hormona produjera ovarios poliquísticos, la mayoría de los investigadores no han encontrado esta respuesta. De Francia han llegado recientemente informes sobre la alarmante producción de grandes ovarios luteinizados después del €mpleo de esta hormona; para mayor información se pueden consultar los informes de BECLERE (Presse med. 68: 31-32, Jan. 9, 1960), RAYMOND et al. (Presse med. 67: 1637, 1959), y MULLER y BAEDER (Compt. rend. Soc. franc. gynec. 29: 371, 1959). Estos autores han prevenido sobre el uso de las grandes dosis y recomiendan el empleo de las pequeñas, esencialmente del mismo orden que fueron empleadas en el esquema original de HAMBLEN en la terapia cíclica 1-2 gonadotrópica. Por otra parte, RYDBERG y sus colaboradores, por muchos años han obtenido buenos resultados empleando esta hormona en altas dosis, pero recientemente RYDBERG (Klin. Fortschr. gynak. pp.: 364-383, 1954) ha recomendado el uso de dosis moderadas para evitar la producción de ovarios poliquísticos. RYDBERG y sus colegas continúan pensando en que la ovulación puede ser inducida con EG y CG administradas cíclica- 
mente y también en que la CG aislada puede inducir ovulaciones en algunas mujeres con ciclos anovulatorics asociados a profusas hemorragias uterinas.

\section{CASUISTICA}

Deseamos poner al día este informe con los datos obtenidos de las historias de cuatro pacientes que recilieron tratamiento gonadotrópico durante los últimos cuatro meses del año 1960: tres pacientes recibieron cada una un ciclo de gonadotropina equina (EG) y gonadotropina coriónica (CG) ; una paciente recibió dos ciclos de gonadotropina pituitaria humana hormona folículo-estimulante (HP-FSH) y gonadotropina coriónica (CG).

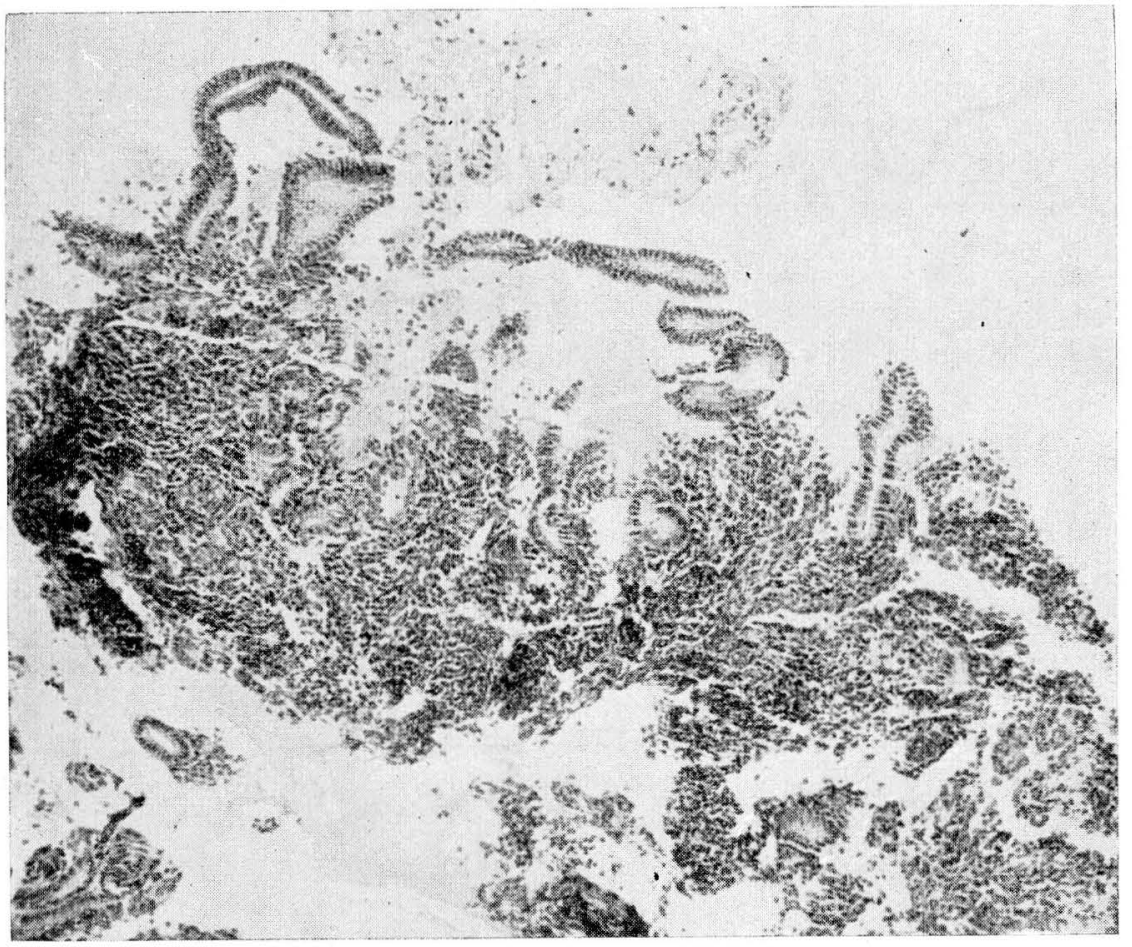

FIGURA 1a - CASO 1 - Biopsia: endometrio hipoestrogénico a la terminación del tratamiento con EG 2.000 U.I. cada 2 días, por 10 días de duración.

\section{CASO 1}

Datos Clínicos: O. S., F-04123, mujer de 20 años de edad, de estatura alta. hipopubescente y quien no había presentado la menarquia. Los genitales ex- 
ternos e internos eran juveniles; tenía ligero desarrollo de los senos y el vello genital era escaso.

Datos significatives de laboratorio: Los frotis bucales resultaron cromatín positivo. El endometrio obtenido por biopsia era hipoestrogénico. Los campos visuales y la radiografía del cráneo eran normales. La iodoproteinemia era de

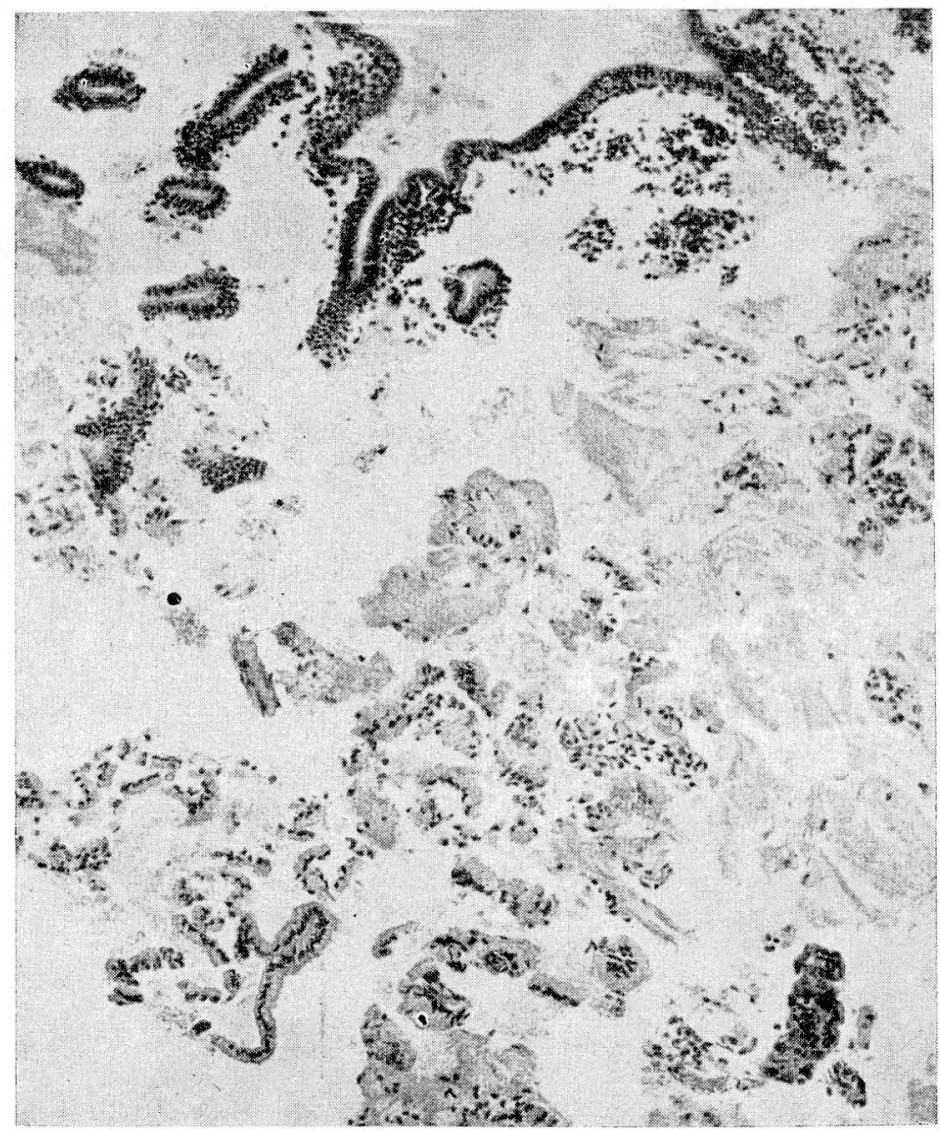

FIGURA 1b - CASO 1 - Biopsia: endometrio hipoestrogénico obtenido 6 días después de practicada la biopsia mostrada en la Figura la y siguiente a las primeras 3 dosis de HCG.

7.2 mcg.\% y el colesterol $355 \mathrm{mg} \%$. Los 17-ketosteroides urinarios $8.4 \mathrm{mg} . / 24$ horas y los 17-hidroxicorticosteroides urinarios $2.6 \mathrm{mg} . / 24$ horas. La determinación de las gonadotropinas urinarias que se practicó en dos ocasiones dio un resultadc de menos de $1 \mathrm{RUU} / 24$ horas (Unidad uterina rata), oscilando los valores normales entre 2 y $10 \mathrm{RUU} / 24$ horas.

Tratamiento: La paciente respondió en numerosas ocasiones con hemorragía uterina cuando se suspendía el tratamiento cíclico con estrógeno y pro- 


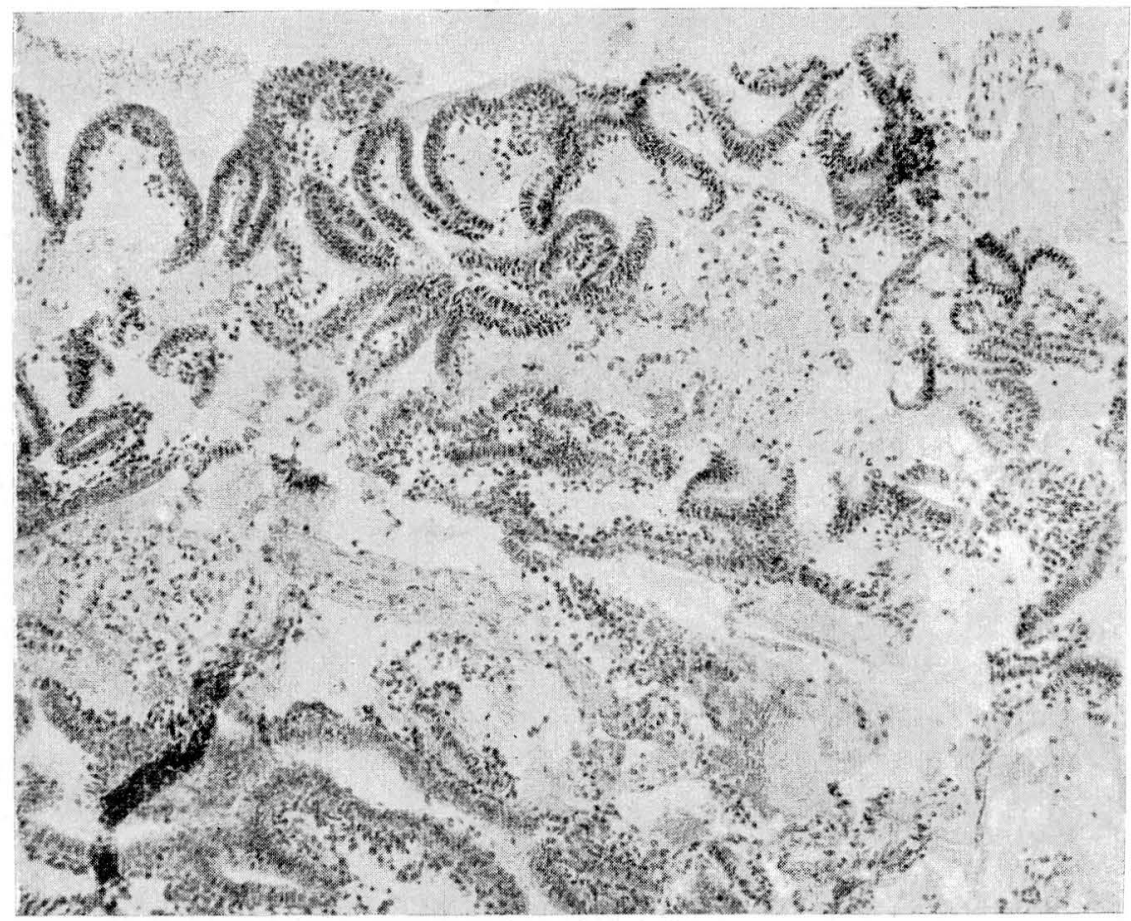

FIGURA 1c - CASO 1 - Biopsia: endometrio hipoestrogénico, tomado 8 días después de practicada la biopsia mostrada en la Figura $1 \mathrm{~b}$ y después de la terminación de las series de terapia gonadotrópica.

gesterona. En noviembre de 1960, la paciente recibió una serie de tratamiento cíclico 1-2 gonadotrópico que se administró en la siguiente forma: EG 2.000 U.I. cada 2 días por espacio de 10 días y subsecuentemente HCG cada 2 días y por espacio de 1 0días en dosis individuales de $3.0003 .000,1.500,1.500$, y 1.000 U.I. respectivamente.

Resultados: No hubo respuesta a este tratamiento gonadotrópico juzgada por la ausencia de aumento del tamaño de los ovarios, ausencia de cambios en la citología vaginal, en la temperatura basal del cuerpo y en el moco cervical. No se presentó hemorragia uterina y el resultado de las biopsias endometriales practicadas en tres ocasiones, durante $y$ al terminar el tratamiento, fue de endometrio hipoestrogénico (figuras 1a, 1b, 1c). 


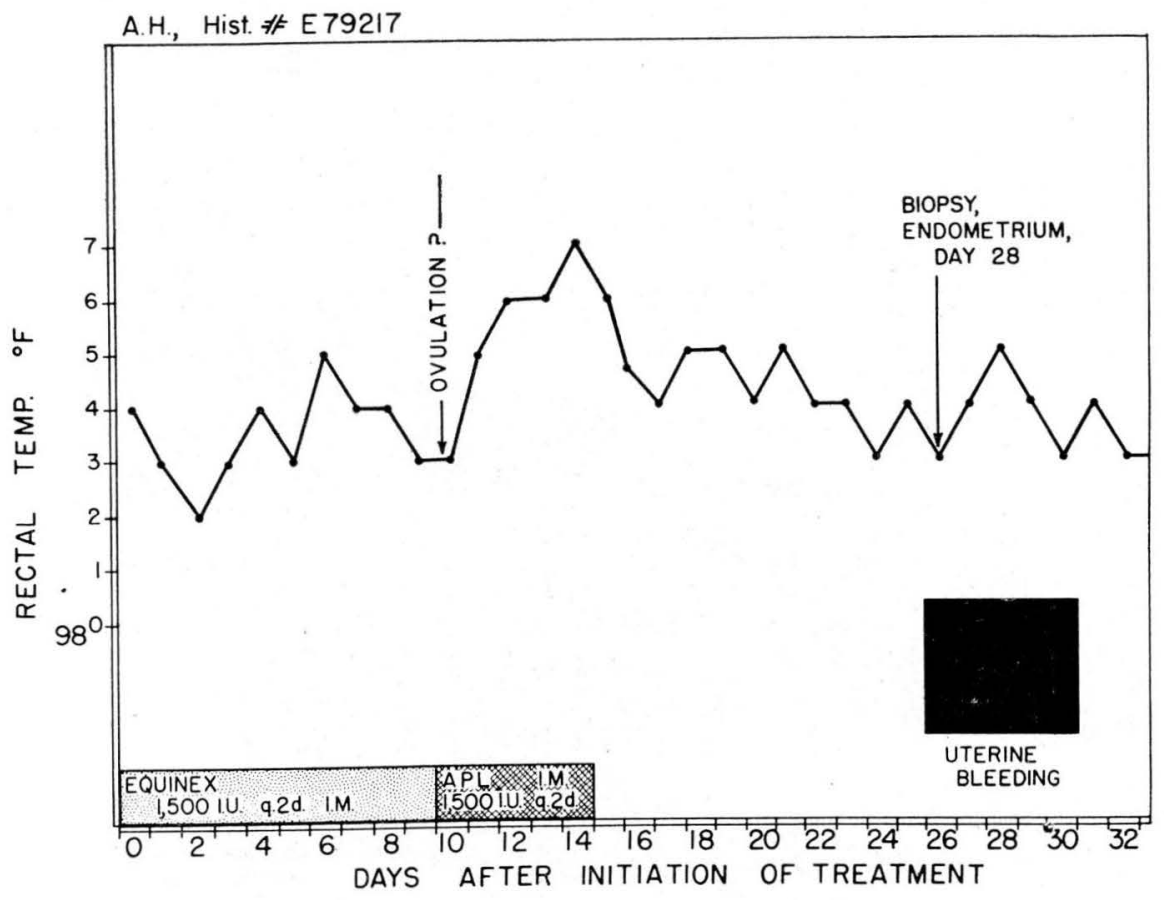

FIGURA 2 - CASO 2 - Detalles del tratamiento y sus respuestas.

\section{CASO 2}

Datos Clínicos: A. H., E-79217, mujer de 26 años, quien había sufrido de hemorragias uterinas profusas y demasiado frecuentes de tipo estrogénico, por un período de 30 meses. Había recibido tratamiento cíclico con estrógeno y progesterona con respuesta poco satisfactoria. La paciente presentaba buena estrogenización y tenía senos, útero y ovarios de caracteres normales. 


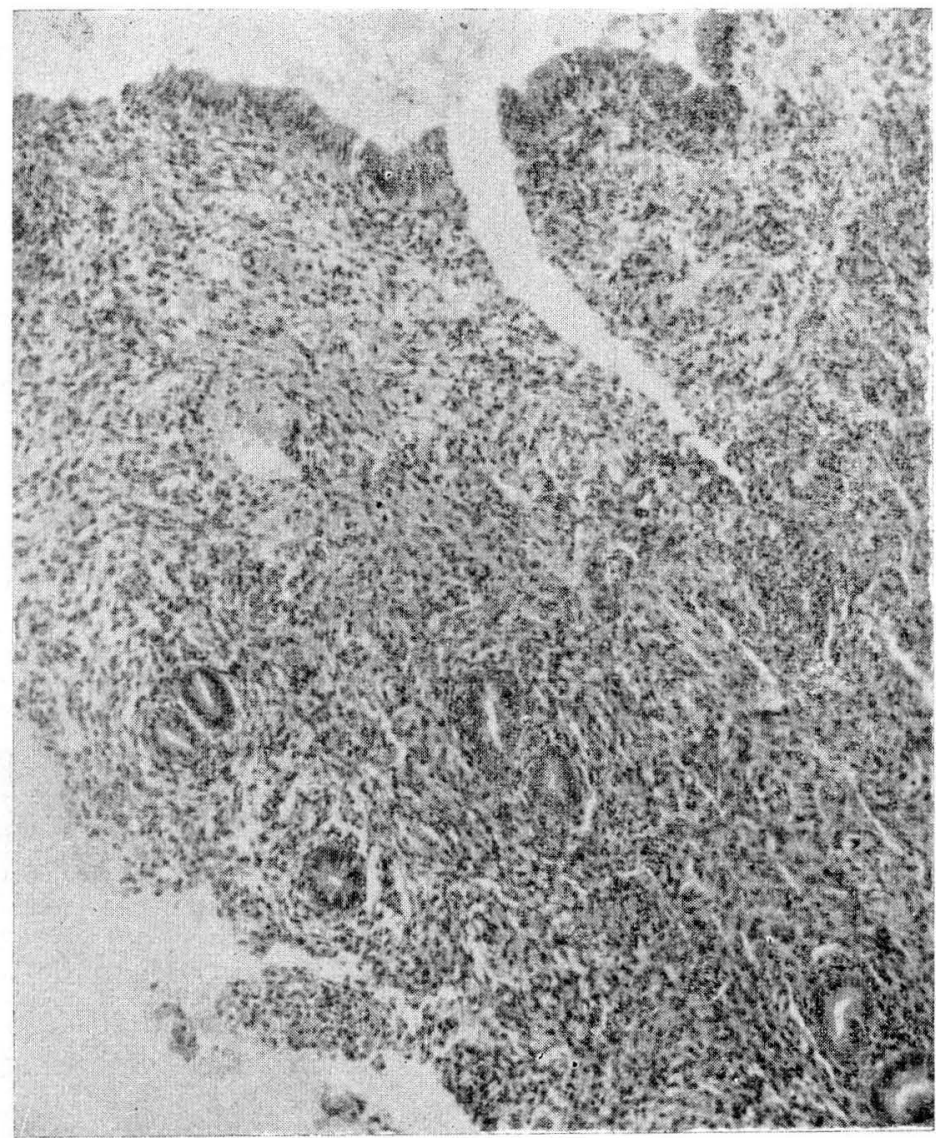

FIGURA 2a - CASO 2 - Biopsia: endometrio estrogénico tardío obtenido al comenzar el período e inmediatamente antes al ciclo de terapia gonadotrópica.

Datos significativos de laboratorio: La hemoglobina era de $11.6 \mathrm{gm}$. y el mecanismo de coagulación sanguíneo era normal. Se practicó un histerograma, encontrándose normal. Se practicaron también repetidas biopsias endometriales a la iniciación de la hemorragia uterina y una inmediatamente antes de comenzar el tratamiento gonadotrópico que dieron por resultado, endometrio de tipo estrogénico. 


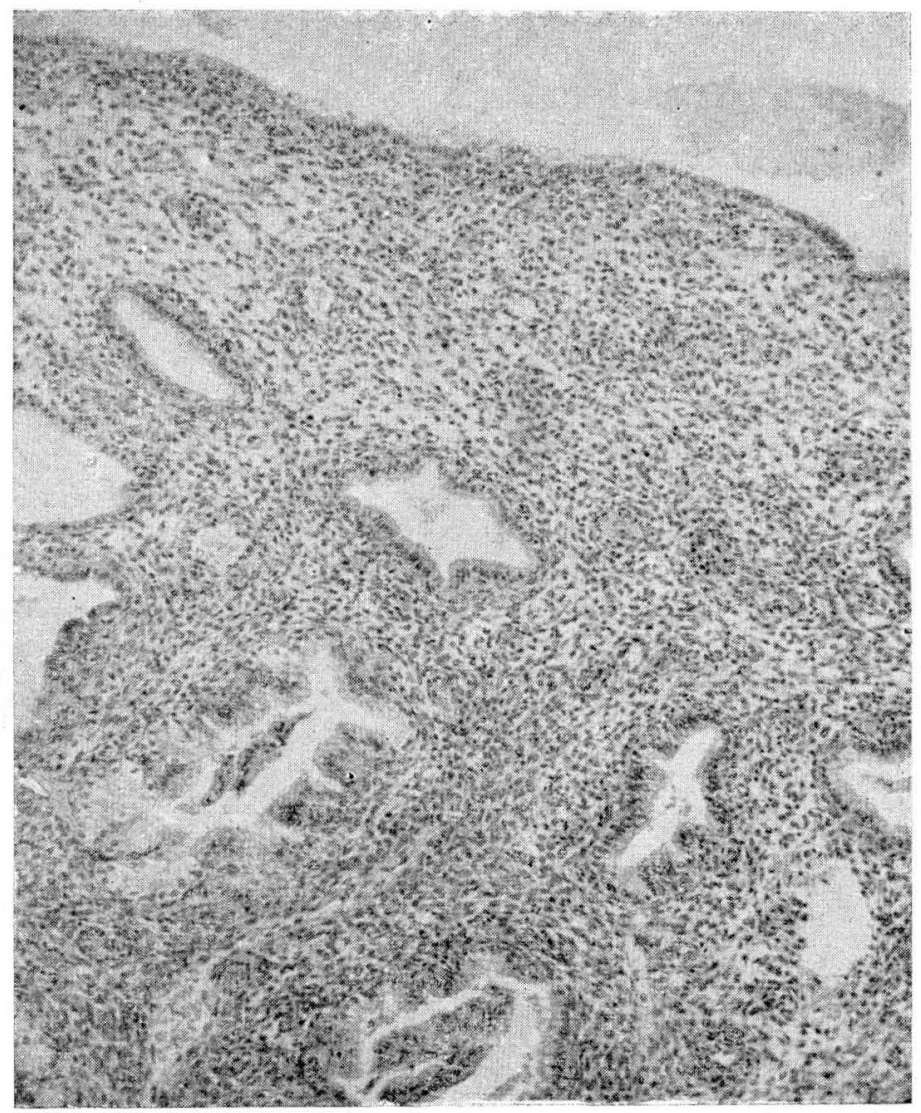

FIGURA 2b - CASO 2 - Biopsia : endometrio progestacional en el día 28, obtenido al comenzar el período y 12 días después de la última inyección de HCG.

Tratamiento: La paciente recibió un ciclo de tratamiento 1-2 gonadotrópico, empleando EG y CG. Los detalles del tratamiento aparecen en la figura 2.

Resultados: Ovulación inducida con el tratamiento. Para evidenciar esta respuesta, véanse las figuras $2,2 \mathrm{a}$ y $2 \mathrm{~b}$. 


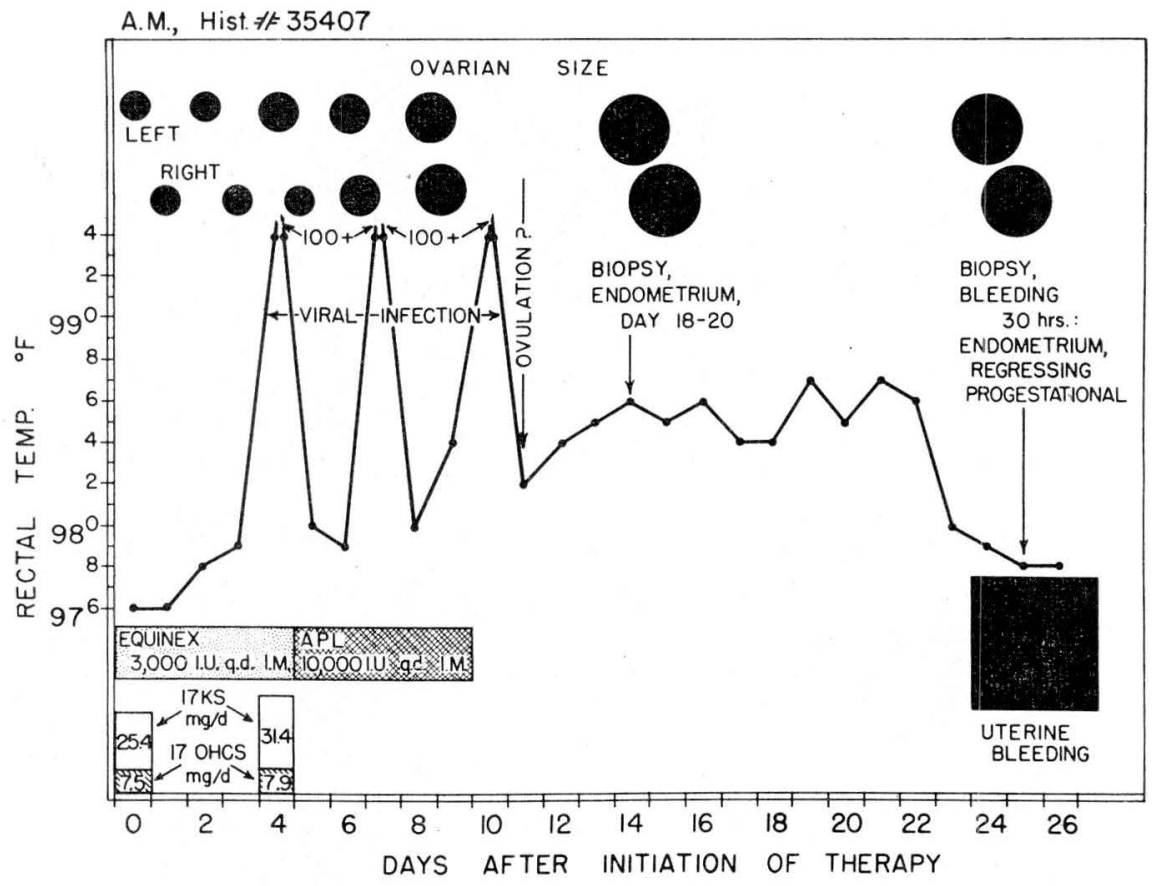

FIGURA 3 - CASO 3 - Detalles del tratamiento y sus respuestas.

\section{CASO 3}

Datos clínicos: A. M., F-35407, mujer de 23 años, quien había tenido la menarquia a la edad de 13 años y posteriormente presentó períodos escasos e infrecuentes por espacio de 5 años y amenorrea durante los últimos 5 años. La paciente deseaba tener descendencia. Aparecía bien estrogenizada aunqu el útero era ligeramente pequeño (histerometría: 2 pulgadas). Los ovarios eran de tamaño normal. Presentaba excesivo desarrollo del vello sobre el labio superior, línea alba y nalgas. La paciente había sido examinada y tratada en Boston, Massachussets, antes de venir a consultar a Duke University Medical Center. 


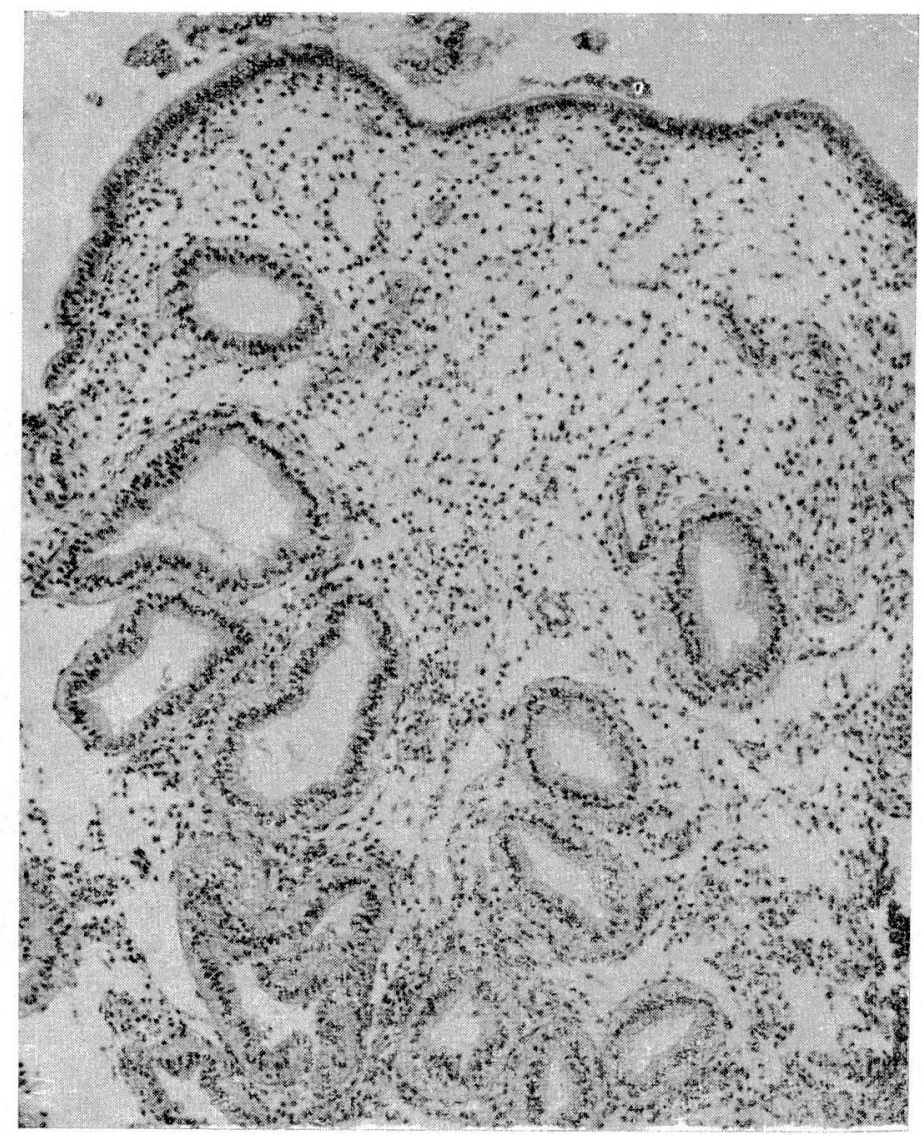

FIGURA 3 a - CASO 3 - Biopsia: endometrio progestacional en el día 18 20 , obsenido de la paciente sin hemorragia y 5 días después de la última inyección de HCG.

Se intentó hacer un diagnóstico del llamado síndrome STEIN-LEVENTHAL o también de una hiperplasia adreno-cortical con mínima virilización.

Datos significativos de laboratorio: Las determinaciones practicadas en Boston, de los 17-ketosteroides urinarios se encontraron que fluctuaban entre 7.3 a $24.6 \mathrm{mg}$./24 horas. Nuestros hallazgos para los 17 ketosteroides urinarios así como los de las biopsias endometriales practicadas, son registrados en las figuras 3 , 3a y $3 \mathrm{~b}$.

Tratamiento: Mientras se encontraba en Boston, la paciente recibió 2 ciclos de tratamiento 1-2 gonadotrópico, empleando HP-FSH y HCG. No hubo respuestas positivas a estos ciclos de tratamiento en cuanto a la ausencia de cambios en la curva de la temperatura basal del cuerpo, en la excreción del pregnandiol urinario o en el tamaño de los ovarios. La paciente no presentó hemorragia uterina y biopsias endometriales no fueron tomadas en Boston. 


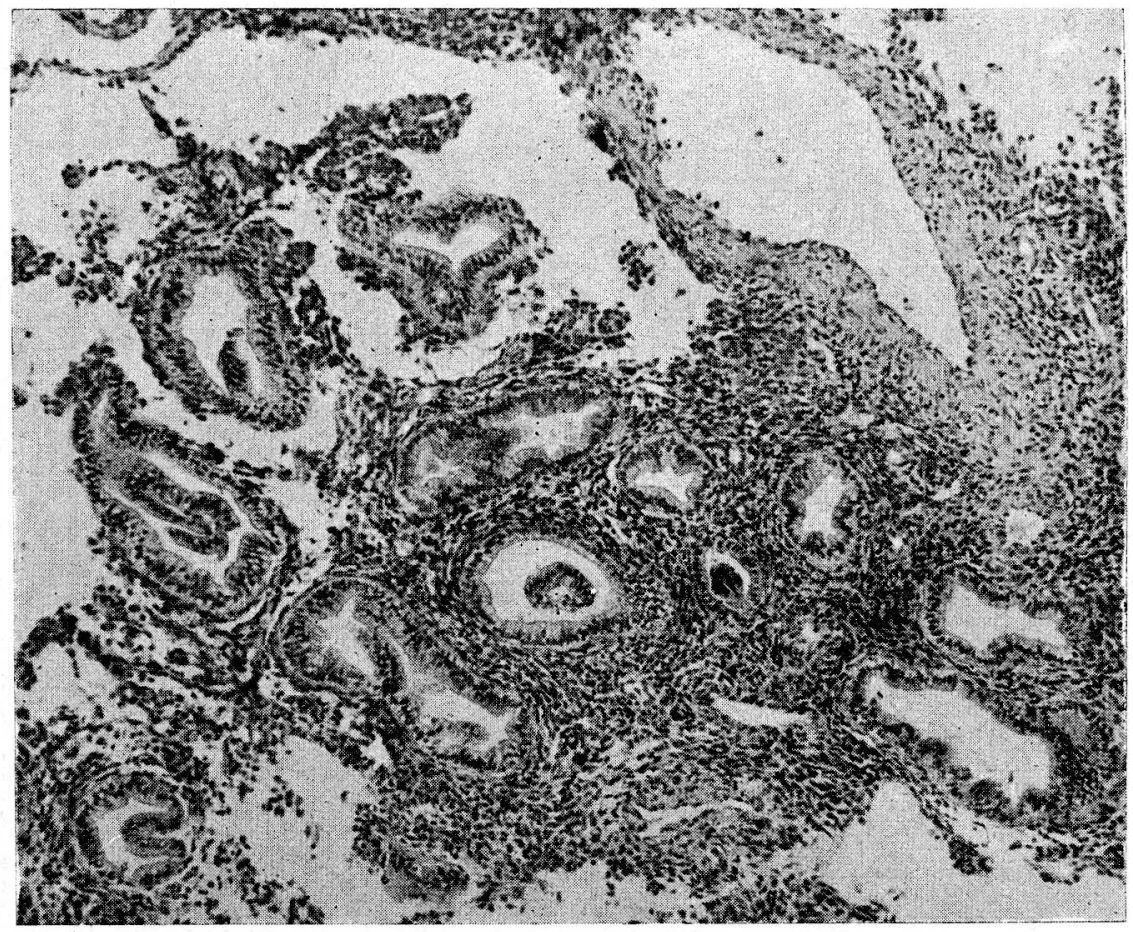

FIGURA 3b - CASO 3 - Biopsia : endometrio progestacional regresivo obtenido de la paciente a las 30 horas cí hemorragia y 16 días después de la última inyección de HCG.

Nosotros administramos a la paciente un ciclo de tratamiento 1-2 gonadotrópico empleando EG y HCG. La paciente presentó hemorragia uterina al terminar el tratamiento. Para los detalles del mismo así como de las respuestas específicas, véanse figuras 3 , 3a y $3 \mathrm{~b}$.

Resuitados: Nosotros juzgamos que la paciente había ovulado y observamos un marcado aumento del tamaño ovárico. Para los detalles, véanse figuras 3 , 3a y $3 \mathrm{~b}$. 


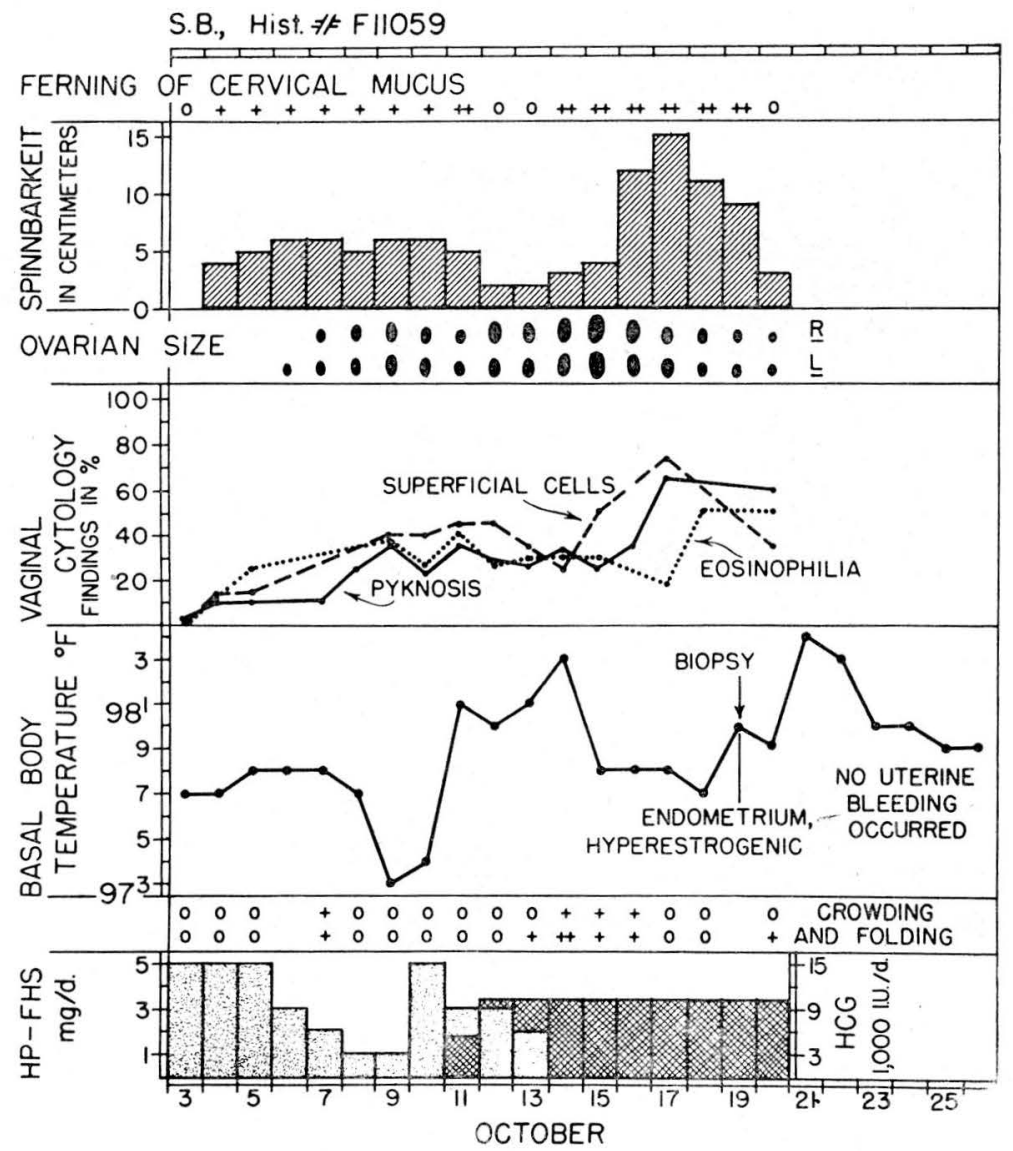

FIGURA 4 - CASO 4 - Detalles del primer ciclo de terapia gonadotrópica y sus respuestas.

\section{CASO 4}

Datos clínicos: S. B., F-11059, mujer de 28 años de edad con un típico síndrome de SHEEHAN de 22 meses de evolución. Presentaba decaimiento general, vejez prematura, entraba en confusión mental fácilmente, tenía piel amarillenta y áspera, alguna alopecia y debilidad muscular generalizada con una fase de relajación lenta; había también atrofia de los senos y de los genitales externos e internos; los ovarios además, eran pequeños. 


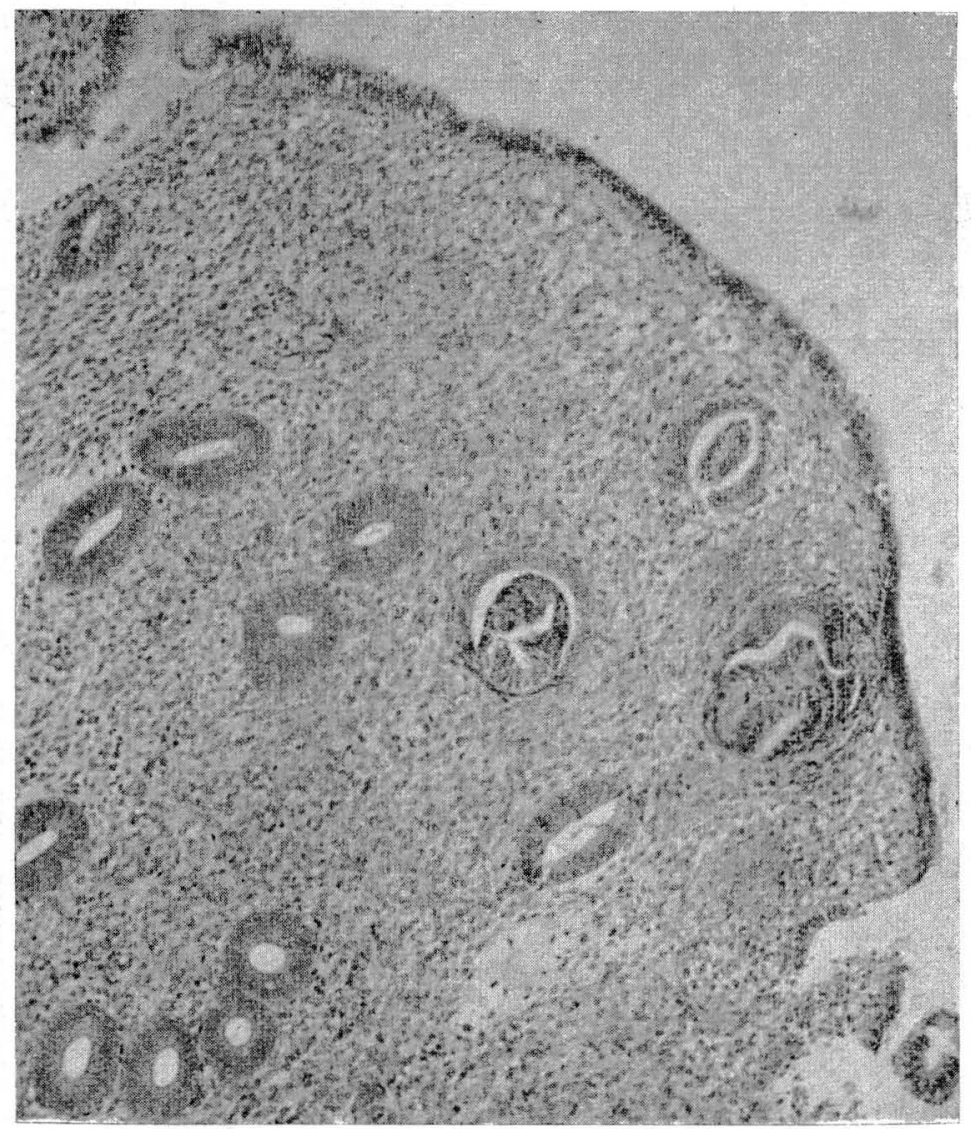

FIGURA 4a - CASO 4 - Biopsia: endometrio hiperestrogénico siguiente al
primer ciclo de terapia gonadotrópica; la paciente no tuvo período.

Datos significativos de laboratorio: Estos informes eran confirmativos del diagnóstico de panhipopituitarismo. La determinación de las gonadotropinas urinarias practicadas en dos ocasiones, dió como resultado, menos de 1 RUU/ 24 horas, fluctuando los valores normales entre 2 y 10 RUU/24 horas. Los 17ketosteroides urinarios eran de $3.3 \mathrm{mg}$. $/ 24$ horas y los 17 -hidroxicorticoides urinarios eran de $2.3 \mathrm{mg} . / 24$ horas. Las respuestas al estímulo suprarrenal y tiroideo por medio de las hormonas ACTH y TSH, eran normales. 


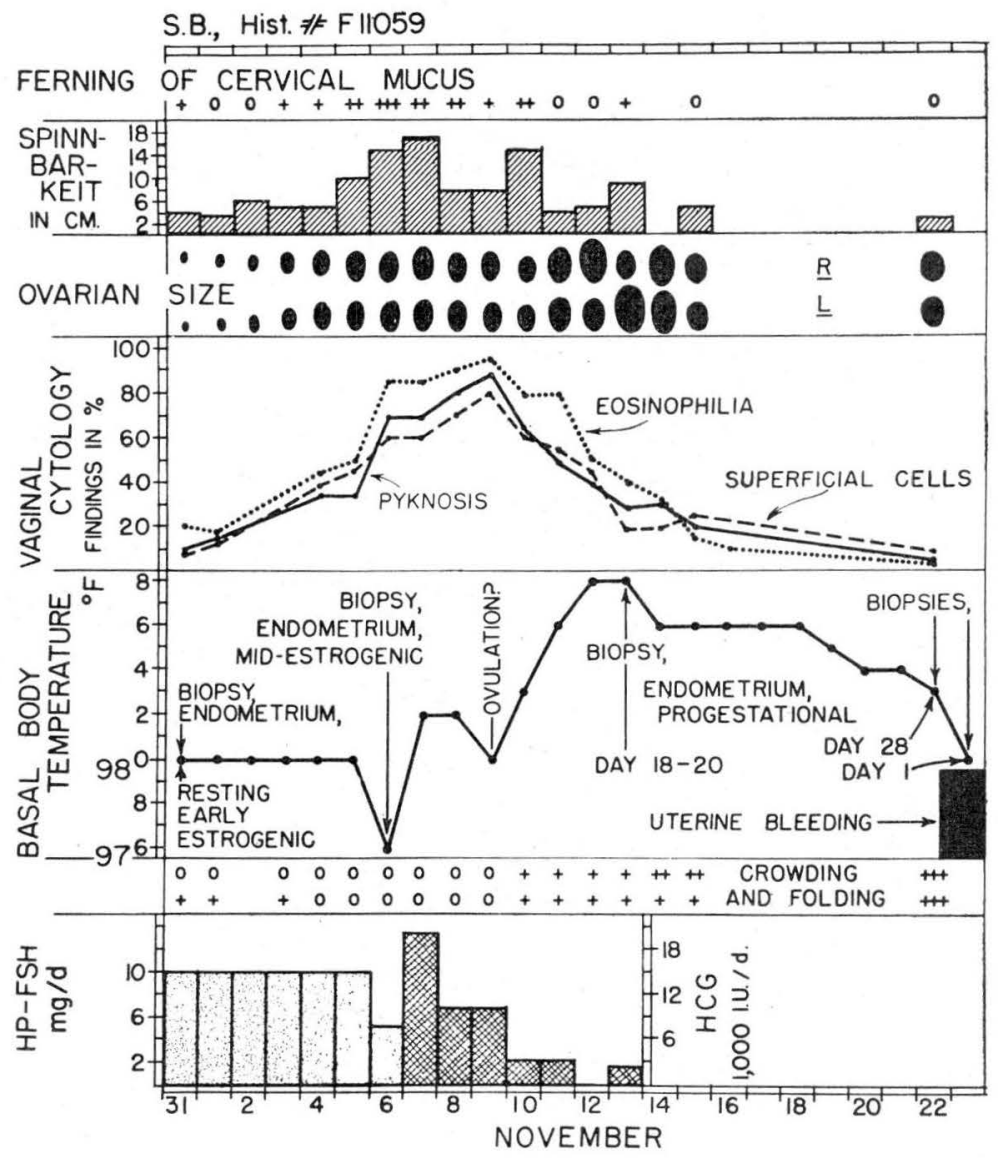

FIGURA 5 - CASO 4 - Detalles del segundo ciclo de terapia gonadotrópica y sus respuestas.

Tratamiento: El tratamiento preliminar y básico de la paciente incluyó Cortisona $25 \mathrm{mg}$. diarios, tiroides desecado U. S. P. $120 \mathrm{mg}$. diarios y tanato de pitresín en aceite para la diabetes insipidus. La paciente recibió también numerosos tratamientos cíclicos con estrógeno y progesterona habiendo respondido con hemorragia uterina cuando se suspendía la administración hormonal. 


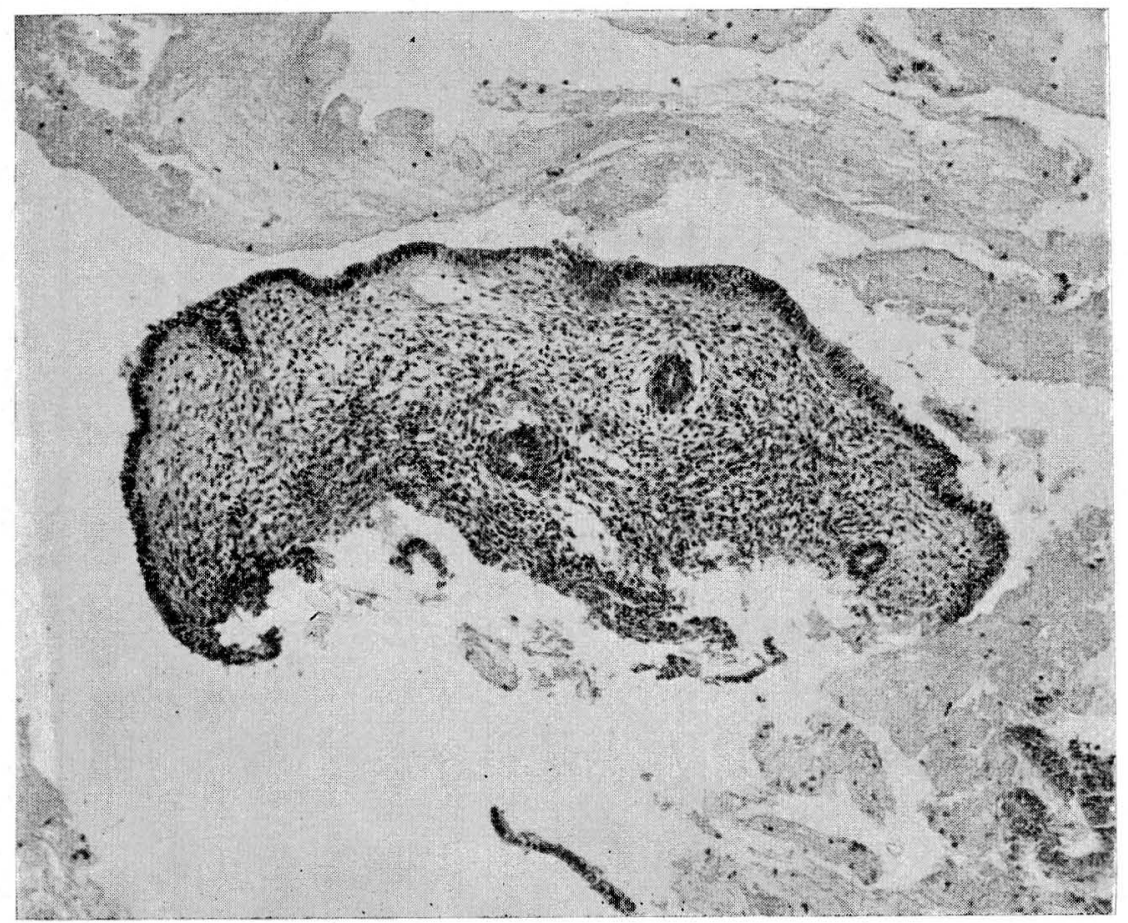

FIGURA 5a - CASO 4 - Biopsia: endometrio estrogénico temprano inactivo, obtenido 11 días después de completar el primer ciclo de terapia gonadotrópica e inmediatamente antes de comenzar el segundo ciclo.

A esta enferma se le administraron 2 ciclos de tratamiento 1-2 gonadotrópico, empleando HP-FSH y HCG. Para los detalles de este tratamiento y sus respuestas específicas véanse figuras $4,4 a, 5,5 a, 5 b, 5 c, 5 d, 5 e$. Antes y durante la administración del segundo ciclo gonadotrópico, se agregó al tratamiento básico, Cytomel en dosis de $25 \mathrm{mg}$. diarios, para asegurar una más completa función tiroidea. 


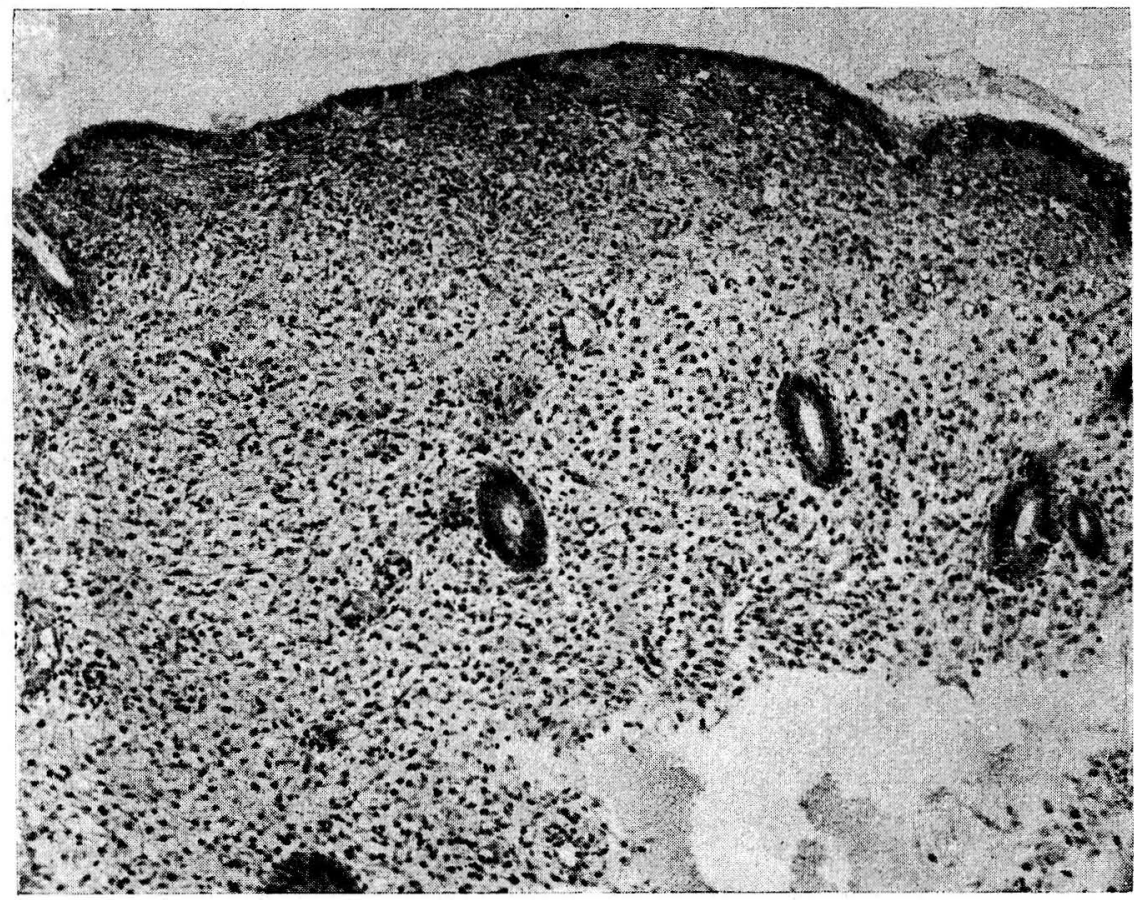

FIGURA 5b - CASO 4 - Biopsia : endometrio en la mitad de la fase estrogénica, obtenido inmediatamente después de la administración de $65 \mathrm{mg}$. de HP.FSH, por espacio de 6 días, como parte del segundo ciclo de tratamiento.

Resultado: No ocurrió ovulación durante el primer ciclo de terapia gonadotrópica. La ovulación seguida de hemorragia uterina apareció en el segundo ciclo de tratamiento. Para una mejor información sobre las respuestas a estos dos ciclos de tratamiento; véanse las figuras $4,4 a, 5,5 a, 5 b, 5 c, 1 d, 5 e$. Durante las dos administraciones de HP-FSH, los valores de gonadotropinas urinarias ascendieron a $6 \mathrm{RUU} / 24$ horas, es decir, que estos hallazgos resultaron dentro de los límites normales. 


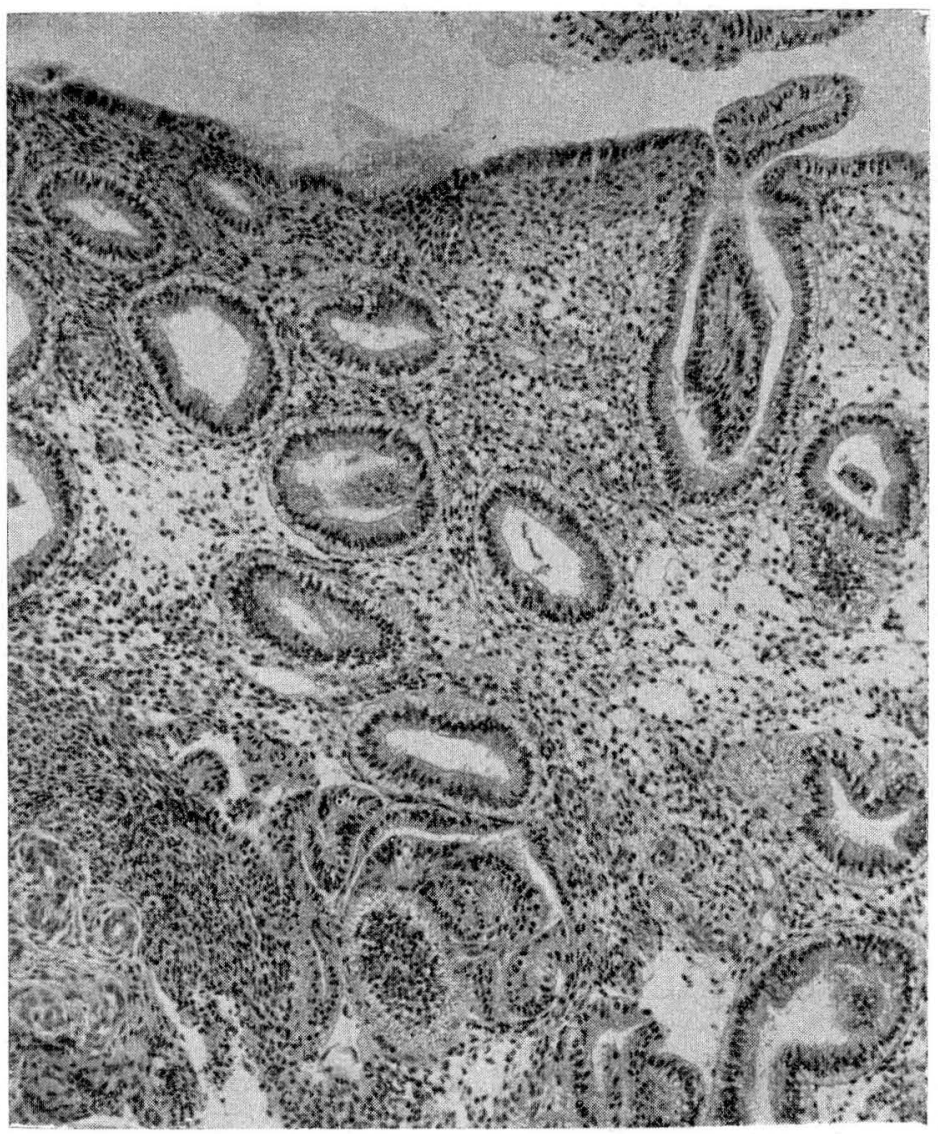

FIGURA 5c - CASO 4 - Biopsia: endometrio progestacional, en el día 1820 , obtenido inmediatamente después de completar el segundo ciclo de terapia gonadotrópica, comprendiendo HP-FSH y HCG. 
Vol. XII

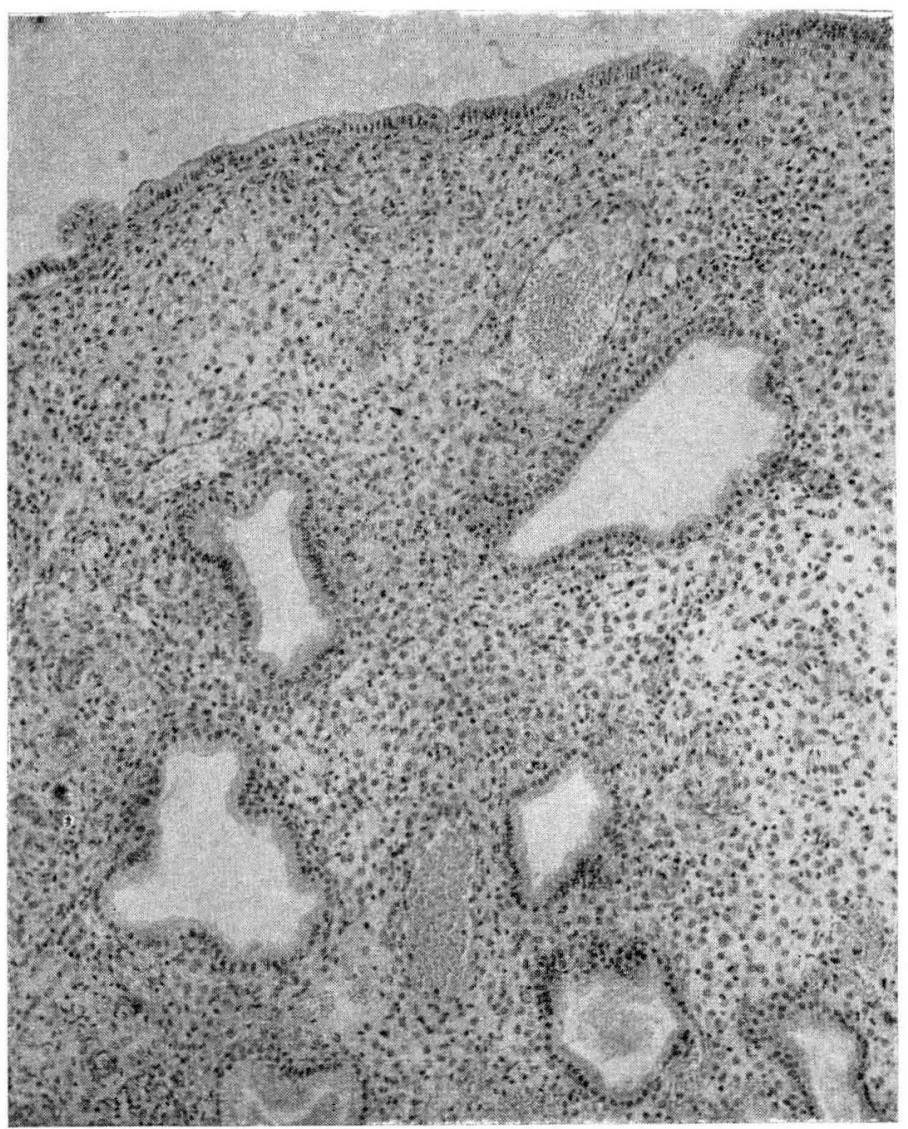

FIGURA 5d - CASO 4 - Biopsia: endometrio progestacional en el día 28 (paciente sin hemorragia). obtenido 9 días después de la última inyección del segundo ciclo de terapia gonadotrópica. 


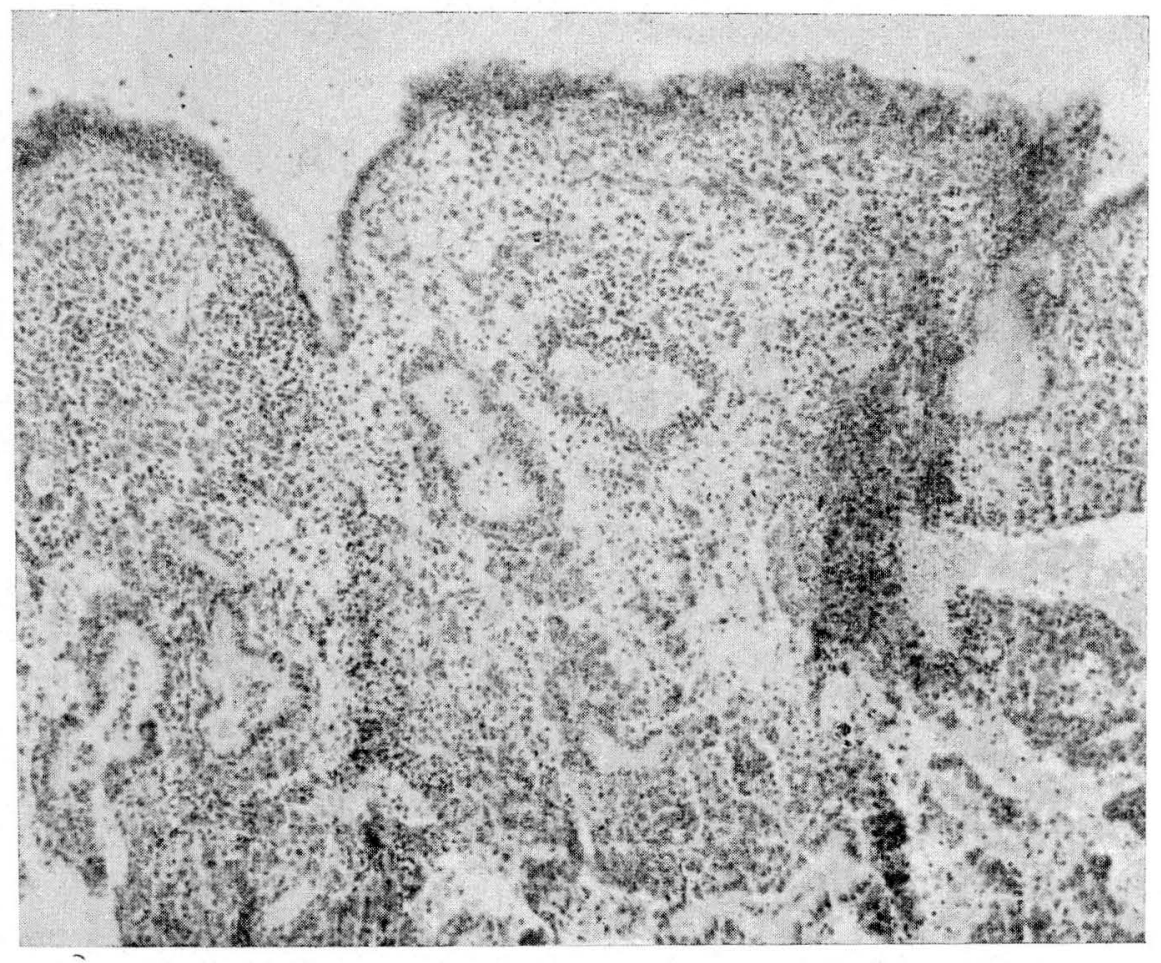

FIGURA 5e - CASO 4 - Biopsia: endometrio menstrual día 1 (paciente con hemorragia de 30 horas), obtenido 10 días después de la última inyección del segundo ciclo de terapia gonadotrópica. 


\section{COMENTARIO}

Los cuatro casos registrados indican que la ovulación puede ser inducida con el tratamiento cíclico 1-2 gonadotrópico, ya sea por el empleo de EG y CG o por el empleo de HP-FSH y CG.

La falta de respuesta en el caso 1, está de acuerdo con la pasada experiencia: la mayoría de las pacientes con apubescencia 0 hipopubescencia, quienes nunca han tenido menstruación, aun cuando ellas tengan hipogonadotropuria, han fallado en responder a este tratamiento. Muchos investigadores han hecho estas observaciones.

También varios de ellos ha reportado respuestas positivas en pacientes que sufren de ciclos anovulatorios con hemorragias de tipo estrogénico, como es el caso de la paciente 2.

La respuesta positiva encontrada en el caso 3 fue inesperada. Esta paciente parecía ser buena candidata para tratamiento con cortisona. El extraño aumento de tamaño de sus ovarios podría sugerir que la hiperplasia adrenocortical predispone a esta respuesta.

Muchos observadores han reportado que pacientes que sufren un síndrome de SHEEHAN, responden mejor a la terapia gonadotrópica. Sin embargo vale la pena notar que la favorable respuesta en el Caso 4, ocurrió cuando se efectuó un mejor control del hipotiroidismo de la paciente.

Es nuestra opinión que, actualmente hay buenos fundamentos para reconsiderar la efectividad de la terapéutica gonadotrópica en los estados anovulatorios.

\section{RESUMEN}

Se ha revisado el valor de los conceptos actuales sobre la acción de la terapéutica gonadotrópica en los estados anovulatorios.

Se ha informado sobre las respuestas de cuatro pacientes consecutivas que recibieron tratamiento cíclico 1-2 gonadotrópico con gonadotropina equina (EG) y gonadotropina coriónica (CG) y un caso de los cuatro mencionados que recibió gonadotropina pituitaria humana-hormona folículoestimulante (HP-FSH) y gonadotropina coriónica (CG). El tratamiento indujo la ovulación en tres de estas cuatro pacientes. Se concluye que, una reva- 
loración acerca de la bondad de la terapia gonadotrópica en los estados anovulatorios, es justificada y que perfeccionamientos tanto en los diagnósticos de niveles endocrinos, como en los esquemas de dosis terapéutica, pueden permitir el tratamiento satisfactorio de muchas pacientes con insuficiencia ovárica hipogonadotrópica.

\section{AGRADECIMIENTOS}

La EG ("Equinex") y la CG ("APL") fueron generosamente suministradas por el Dr. JOHN B. JEWELL, Laboratorios Ayerst, New York, N. Y.

La HP-FSH fue suministrada por Merck \& Company, Rahwey, New Jersey, por el amable intermedio del Dr. F. L. ENGEL, Director de la División de Endocrinología, Duke University Medical Center. El Dr. W. KENNETH CUYLER, jefe de los laboratorios de citología del Departamento de Obstetricia y Ginecología, hizo los estudios citológicos del Caso 4. El Dr. JACK KOSTYO del Departamento de Fisiología fue muy servicial en seguir la paciente reportada en el Caso 4. El Sr. CARL BISHOP del Departamento de Patología, bondadosamente preparó las microfotografías empleadas en el trabajo. Estamos muy agradecidos también con la Sra. FRANCES J. LUNAS del Departamento de Ilustración Médica, por las 4 gráficas preparadas para la demostración de los datos clínicos de los casos 2, 3 y 4 . 